EĖSTI NSV TEADUSTE AKADEEMIA TOIMETISED. 25, KÖIDE FUUSIKA * MATEMAATIKA. 1976, NR. 3

ИЗВЕСТИЯ АКАДЕМИИ НАУК ЭСТОНСКОИ ССР. ТОМ 25 ФИЗИКА * МАТЕМАТИКА. 1976, № 3

П. $О Я$

\title{
ОБ УСТОЙЧИВОСТИ МЕТОДА ГАЛЕРКИНА ДЛЯ ЭВОЛЮЦИОННЫХ УРАВНЕНИЙ
}

Устойчивость метода Галеркина для эволюционных уравнений уже исследована в некоторых работах $\left[{ }^{1-8}\right]$. Мы исследуем ее в условиях Лионса [ $\left.{ }^{9}\right]$ при нормах, для которых доказана $\left[{ }^{10}\right]$ сходимость приближенных решений. Разность возмущенного и невозмущенного галеркинских приближений оценим двусторонне через возмущения, нормы решений приближенных задач и наименьшие собственные значения матриц Грама, образованных из координатных элементов. Полученные оценки вытекают из того, что приближенные задачи задают равномерные изоморфизмы между пространствами решений и пространствами начальных данных. Иs оценок следует необходимое и достаточное условие устойчивости, характеризующее координатную систему.

\section{1. Обозначения и предположения}

Пусть $V$ и $H$ - сепарабельные вещественные гильбертовы пространства, $V$ непрерывно вложено в $H$ и плотно в нем. Тогда

$$
V \subset H \subset V^{\prime},
$$

где через $V^{\prime}$ обозначено пространство, двойственное к $V$. Если $f \in V^{\prime}$ и $v \in V$, то значение функционала $f$ на $v$, обозначенное через $(f, v)$, совпадает со скалярным произведением в $H$ в случае $f \in H$. Обозначим

тогда

$$
\mathfrak{B}=L^{2}(0, T ; V), \quad \mathfrak{S}=L^{2}(0, T ; H) ;
$$

$$
\mathfrak{B}^{\prime}=L^{2}\left(0, T ; V^{\prime}\right), \quad \mathfrak{B} \subset \mathfrak{S} \subset \mathfrak{B}^{\prime} .
$$

Пусть задано семейство непрерывных линейных операторов $A(t) \in \mathcal{L}\left(V, V^{\prime}\right), t \in[0, T]$. Наложим на него следующие условия [ $\left.{ }^{9}\right]:$ $\forall u, v \in V$ функция $t \rightarrow(A(t) u, v)$ измерима и

$$
(A(t) u, v) \leqslant M\|u\|_{v}\|v\|_{v} \quad \forall u, v \in V ;
$$

существует $\alpha>0$ такое, что

$$
(A(t) v, v) \geqslant \alpha\|v\|_{V}^{2} \quad \forall v \in V .
$$

Рассмотрим задачу

$$
u^{\prime}(t)+A(t) u(t)=f(t), \quad u(0)=\xi,
$$


где $f \in \mathfrak{Y}^{\prime}$ и $\xi \in H-$ заданы, $u^{\prime}=d u / d t$ обозначает производную в смысле распределений. Известно (см. [ $\left.{ }^{9}\right]$, с. $\left.260,268-269\right)$, что задача (1.3) имеет единственное решение и задает изоморфизм из пространства $\mathfrak{B} \cap D\left(d / d t ; \mathfrak{S}^{\prime}\right)=\left\{v \mid v \in \mathfrak{B}, v^{\prime} \in \mathfrak{S}^{\prime}\right\}$ на $\mathfrak{S}^{\prime} \times H$. При этом пространство $\mathfrak{B} \cap D\left(d / d t ; \mathfrak{J}^{\prime}\right)$ снабжается нормой

$$
\|v\|_{\mathfrak{B} \cap D\left(d / d t ; \mathfrak{B}^{\prime}\right)}=\left(\|v\|_{\mathfrak{B}^{2}}^{2}+\left\|v^{\prime}\right\|_{\mathfrak{B}^{\prime}}^{2}\right)^{1 / 2} .
$$

Выберем в пространстве $V$ полную линейно независимую систему $\left\{w_{k}\right\}_{1}^{\infty}$ (она полна также в пространствах $H$ и $V^{\prime}$ ). Линейные оболочки систем $\left\{w_{k}\right\}_{1}^{n}$, снабженные нормами из $V$ и $H$, обозначим через $V_{n}$ и $H_{n}$, а если будем рассматривать их как пространства, двойственные к $V_{n}$, - через $V_{n}^{\prime}$. Итак,

$$
\|v\|_{V_{n}^{\prime}}=\sup _{x \in V_{n},\left\|x_{\|}\right\|_{v}=1}(v, x) \leqslant\|v\|_{V^{\prime}}, \quad v \in V_{n}^{\prime} .
$$

Пусть $P_{n}-$ ортопроектор в $H$, проектирующий на $H_{n}$. Оператор $P_{n}$ ограничен единицей и как оператор из $V^{\prime}$ в $V_{n}{ }^{\prime}$. Расширение $P_{n}$ по непрерывности на $V^{\prime}$ будем также обозначать через $P_{n}$, при этом $\left\|P_{n}\right\|_{V^{\prime} \rightarrow V_{n^{\prime}}} \leqslant 1$. Обозначим также

$$
\mathfrak{S}_{n}=L^{2}\left(0 ; T ; V_{n}\right), \quad \mathfrak{S}_{n}=L^{2}\left(0, T ; H_{n}\right), \quad \mathfrak{B}_{n}{ }^{\prime}=L^{2}\left(0 ; T ; V_{n}{ }^{\prime}\right) .
$$

Рассмотрим еще задачу

$$
u_{n}^{\prime}(t)+P_{n} A(t) u_{n}(t)=P_{n} f(t), \quad u_{n}(0)=P_{n} \xi,
$$

которая имеет при каждом $n$ также единственное решение и задает (равномерный по $n)$ изоморфизм из $\mathfrak{B}_{n} \cap D\left(d / d t ; \mathfrak{B}_{n}^{\prime}\right)$ на $\mathfrak{B}_{n}{ }^{\prime} \times H_{n}$. В частности, величина

$$
\begin{gathered}
c=\sup \left\{\left(\left\|v_{n}\right\|_{\mathfrak{S}_{n^{\prime}}}^{2}+\left\|v_{n}{ }^{\prime}\right\|_{\mathfrak{B}_{n^{\prime}}}^{2}\right)^{1 / 2} \mid v_{n}{ }^{\prime}+P_{n} A v_{n}=f_{n}, v_{n}(0)=\xi_{n},\right. \\
\left.\left\|f_{n}\right\|_{\mathfrak{B}^{\prime}}{ }^{-+}+\left\|\xi_{n}\right\|_{H_{n}}=1, n=1,2, \ldots\right\}
\end{gathered}
$$

конечна. Имеют место следующие сходимости $\left[{ }^{10}\right]$ :

$$
\begin{gathered}
\left(\left\|u_{n}-u\right\|_{\mathfrak{B}}^{2}+\left\|u_{n}{ }^{\prime}-P_{n} u^{\prime}\right\|_{\mathfrak{B}_{n^{\prime}}}^{2}\right)^{1 / 2} \rightarrow 0, \\
\left\|u_{n}-u\right\|_{C([0, T] ; H)} \rightarrow 0 .
\end{gathered}
$$

Естественно возникает вопрос об устойчивости решения задачи (1.4) относительно малых возмущений при вычислении.

Решение $u_{n}(t)$ будем искать в виде

$$
u_{n}(t)=\sum_{k=1}^{n} c_{n k}(t) w_{k}
$$

Задача (1.4) равносильна задаче

$$
\begin{gathered}
\sum_{k=1}^{n}\left(w_{k}, w_{i}\right) c_{n k}{ }^{\prime}(t)+\sum_{k=1}^{n}\left(A(t) w_{k}, w_{i}\right) c_{n k}(t)=\left(f(t), w_{i}\right), \\
\sum_{k=1}^{n}\left(w_{k}, w_{i}\right) c_{n k}(0)=\left(\xi, w_{i}\right), \quad i=1, \ldots, n .
\end{gathered}
$$

Бходящие сюда скалярные произведения вычисляются, как правило, с некоторыми погрешностями, т. е. вместо задачи (1.6) подлежит решению возмущенная задача 


$$
\begin{gathered}
\sum_{k=1}^{n}\left(\left(w_{k}, w_{i}\right)+\gamma_{i k}\right) \tilde{c}_{n k}{ }^{\prime}(t)+\sum_{k=1}^{n}\left(\left(A(t) w_{k}, w_{i}\right)+\alpha_{i k}(t)\right) \tilde{c}_{n k}(t)= \\
=\left(f(t), w_{i}\right)+g_{i}^{(n)}(t), \\
\sum_{k=1}^{n}\left(\left(w_{k}, w_{i}\right)+\gamma_{i k}\right) \tilde{c}_{n k}(0)=\left(\xi, w_{i}\right)+\delta_{i}^{(n)}, i=1, \ldots, n .
\end{gathered}
$$

Вследствие этого получаем вместо $u_{n}$ возмущенное решение

$$
\tilde{u}_{n}(t)=\sum_{k=1}^{n} \tilde{c}_{n k}(t) w_{k}
$$

Введем обозначения

$$
\begin{array}{r}
\Gamma_{n}=\left\{\gamma_{i k}\right\}_{i, k=1}^{n} \quad-\text { симметричная матрица, } \\
\Delta_{n}(t)=\left\{\alpha_{i k}(t)\right\}_{i, k=1}^{n}-\text { измеримая по } t \text { матрица, } \\
g_{n}(t)=\left\{g_{i}^{(n)}(t)\right\}_{i=1}^{n}, \quad \delta_{n}=\left\{\delta_{i}^{(n)}\right\}_{i=1}^{n},
\end{array}
$$

$\Lambda_{n}=\left\{\left(w_{i}, w_{k}\right)\right\}_{i, k=1}^{n}, \quad M_{n}=\left\{\left(w_{i}, w_{k}\right)_{V_{n}{ }^{\prime}}\right\}_{i, k=1}^{n}, \quad N_{n}=\left\{\left(w_{i}, w_{k}\right)_{V}\right\}_{i, k=1}^{n}$.

Матрицы и векторы будем рассматривать как операторы и элементы эвклидова пространства $\mathbf{R}^{n}$; соответствующий смысл придается их нормам.

\section{2. Основные оценки}

Пусть $\lambda_{n}, \mu_{n}, v_{n}$ - наименьшие собственные значения матриц Грама $\Lambda_{n}, M_{n}, N_{n}$ соответственно, число с определено в (1.5).

Т е ор е м. Пусть выполнены условия (1.1) $u$ (1.2).

$1^{\circ}$ Ecлu $\left\|\Gamma_{n}\right\| \leqslant \frac{p}{\sqrt{2} c} \sqrt{\mu_{n}} \sqrt{v_{n}}(p<1),\left\|\Gamma_{n}\right\| \leqslant r \lambda_{n} \quad(r<1)$, $\sup _{t \in[0, T]}\left\|\Delta_{n}(t)\right\| \leqslant \frac{q}{\sqrt{2} c} v_{n}(q<1)$,

то система (1.7) однозначно разрешима и

$$
\begin{aligned}
& \left\|\tilde{u}_{n}-u_{n}\right\| \equiv\left(\left\|\tilde{u}_{n}-u_{n}\right\|_{L^{2}(0, T ; V)}^{2}+\left\|\tilde{u}_{n}{ }^{\prime}-u_{n}{ }^{\prime}\right\|_{L^{2}\left(0, T ; V_{n}\right)}^{2}\right)^{1 / 2} \leqslant \\
& \leqslant \frac{c}{1-\max \{p, q\}}\left(\frac{\left\|u_{n}{ }^{\prime}\right\|_{L^{2}\left(0, T ; V_{n}\right)}}{\sqrt{\mu_{n}} \sqrt{v_{n}}}\left\|\Gamma_{n}\right\|+\right. \\
& +\frac{\left\|u_{n}\right\|_{L^{2}\left(0, T ; V_{n}\right)}}{v_{n}} \sup _{t \in[0, T]}\left\|\Delta_{n}(t)\right\|+\frac{1}{\sqrt{v_{n}}}\left\|g_{n}\right\|_{L^{2}\left(0, T ; \mathrm{R}^{n}\right)}+ \\
& \left.+\frac{\left\|u_{n}(0)\right\|_{H_{n}}}{(1-r) \lambda_{n}}\left\|\Gamma_{n}\right\|+\frac{1}{(1-r) \sqrt{\lambda_{n}}}\left\|\delta_{n}\right\|\right) .
\end{aligned}
$$

$2^{\circ}$ Найдутся не зависящие от $n$ положительные постоянные $c_{1}, \ldots, c_{5}$ такие, что: 
1) если $\Delta_{n}(t)=0, g_{n}(t)=0, \delta_{n}=0$, то существуют $\Gamma_{n} \neq 0$ (со сколь угодно малой нормой) $и u_{n} \neq 0$ такие, что

$$
\left\|\tilde{u}_{n}-u_{n}\right\| \geqslant \frac{c_{1}\left\|u_{n}\right\|}{\sqrt{\mu_{n}} \sqrt{v_{n}}}\left\|\Gamma_{n}\right\| ;
$$

2) если $\Gamma_{n}=0, g_{n}(t)=0, \delta_{n}=0$, то существуют $\Delta_{n}(t) \neq 0$ (со сколь угодно малой нормой) $и u_{n} \neq 0$ такие, что

$$
\left\|\tilde{u}_{n}-u_{n}\right\| \geqslant \frac{c_{2}\left\|u_{n}\right\|}{v_{n}} \sup _{t \in[0, T]}\left\|\Delta_{n}(t)\right\| ;
$$

3) если $\Delta_{n}(t)=0, g_{n}(t)=0, \delta_{n}=0$, то существуют $\Gamma_{n} \neq 0$ (со сколь угодно малой нормой) $и u_{n} \neq 0$ такие, что

$$
\left\|\tilde{u}_{n}-u_{n}\right\| \geqslant \frac{c_{3}\left\|u_{n}(0)\right\|_{H_{n}}}{\lambda_{n}}\left\|\Gamma_{n}\right\| ;
$$

4) если $\Gamma_{n}=0, \Delta_{n}(t)=0, \delta_{n}=0$, то существует $g_{n}(t) \neq 0$ такой, чTO

$$
\left\|\tilde{u}_{n}-u_{n}\right\| \geqslant \frac{c_{4}}{\sqrt{\lambda_{n}}}\left\|g_{n}\right\|_{L^{2}\left(0, T ; \mathbf{R}^{n}\right)} ;
$$
чTO

5) если $\Gamma_{n}=0, \Delta_{n}(t)=0, g_{n}(t)=0$, то существует $\delta_{n} \neq 0$ такой,

$$
\left\|\tilde{u}_{n}-u_{n}\right\| \geqslant \frac{c_{5}}{\sqrt{\lambda_{n}}}\left\|\delta_{n}\right\| .
$$

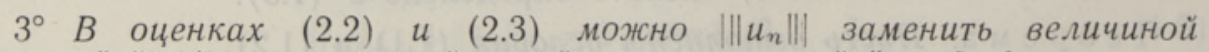
$\left(\|f\|_{\mathfrak{B}^{\prime}}^{2}+\|\xi\|_{H}^{2}\right)^{1 / 2}, \quad a_{в}(2.4)\left\|u_{n}(0)\right\|_{H_{n}}$ величиной $\|\xi\|_{H}$, где f $u \xi-$ некоторые элементы в (1.3) (зависящие от $n$ ).

Д ок а з тельст в о. Введем обозначения

$$
\psi_{n} x=\left(\left(x, w_{1}\right), \ldots,\left(x, w_{n}\right)\right) \quad\left(x \in V_{n}\right), \quad T_{n}=\Lambda_{n}^{-1} \psi_{n} .
$$

Тогда $T_{n} x=\left(c_{1}, \ldots, c_{n}\right) \in \mathrm{R}^{n}$ для $x=\sum_{k=1}^{n} c_{k} w_{k}$, т. е. $T_{n}$ является оператором отделения координат.

Возмущенная задача (1.7) принимает вид

$$
\begin{aligned}
& \tilde{u}_{n}{ }^{\prime}+\psi_{n}^{-1} \Gamma_{n} T_{n} \tilde{u}_{n}{ }^{\prime}+P_{n} A \tilde{u}_{n}+\psi_{n}^{-1} \Delta_{n} T_{n} \tilde{u}_{n}=P_{n} f+\psi_{n}^{-1} g_{n}, \\
& \tilde{u}_{n}(0)+\psi_{n}^{-1} \Gamma_{n} T_{n} \tilde{u}_{n}(0)=P_{n} \xi+\psi_{n}^{-1} \delta_{n} .
\end{aligned}
$$

Для $h_{n}=\tilde{u}_{n}-u_{n}$ имеем

$$
\begin{gathered}
\left(I_{n}+\psi_{n}^{-1} \Gamma_{n} T_{n}\right) h_{n}{ }^{\prime}+\left(P_{n} A+\psi_{n}^{-1} \Delta_{n} T_{n}\right) h_{n}= \\
=\psi_{n}^{-1} g_{n}-\psi_{n}^{-1} \Gamma_{n} T_{n} u_{n}^{\prime}-\psi_{n}^{-1} \Delta_{n} T_{n} u_{n}, \\
\left(I_{n}+\psi_{n}^{-1} \Gamma_{n} T_{n}\right) h_{n}(0)=\psi_{n}^{-1} \delta_{n}-\psi_{n}^{-1} \Gamma_{n} T_{n} u_{n}(0),
\end{gathered}
$$


В [ $\left.{ }^{11}\right]$ (с. 242) показано, что

$$
\left\|\psi_{n}^{-1}\right\|_{\mathbf{R}^{n} \rightarrow H_{n}}=\frac{1}{\sqrt{\lambda_{n}}}, \quad\left\|T_{n}\right\|_{H_{n} \rightarrow \mathrm{R}^{n}}=\frac{1}{\sqrt{\lambda_{n}}} .
$$

Заменив $H_{n}$ пространствами $V_{n}$ и $V_{n}{ }^{\prime}$, получим

Докажем еще равенства

$$
\left\|T_{n}\right\|_{V_{n} \rightarrow \mathrm{R}^{n}}=\frac{1}{\sqrt{v_{n}}}, \quad\left\|T_{n}\right\|_{V_{n}^{\prime} \rightarrow \mathrm{R}^{n}}=\frac{1}{\sqrt{\mu_{n}}} .
$$

$$
\left\|\psi_{n}^{-1}\right\|_{\mathrm{R}^{n} \rightarrow V_{n}^{\prime}}=\frac{1}{\sqrt{v_{n}}}, \quad\left\|\psi_{n}^{-1}\right\|_{\mathrm{R}^{n \rightarrow V_{n}}}=\frac{1}{\sqrt{\mu_{n}}} .
$$

Очевидно, $\quad \psi_{n}^{-1}=T_{n}^{-1} \Lambda_{n}^{-1}$. Таким образом,

$$
\begin{aligned}
\left\|\Psi_{n}^{-1}\right\|_{\mathbf{R}^{n} \rightarrow V_{n}^{\prime}} & =\sup _{\|x\|_{\mathbf{R}^{n}}=1,\|v\|_{V_{n}}=1}\left(T_{n}^{-1} \Lambda_{n}^{-1} x, v\right)= \\
& =\sup _{\|x\|_{\mathrm{R}^{n}}=1,\|v\|_{V_{n}}=1}\left(\sum_{n=1}^{n}\left[\Lambda_{n}^{-1} x\right]_{k} w_{k}, \sum_{i=1}^{n}\left[T_{n} v\right]_{i} w_{i}\right)= \\
& =\sup _{\|x\|_{\mathbf{R}^{n}}=1,\|v\|_{V_{n}}=1}\left(\Lambda_{n}^{-1} x, \Lambda_{n} T_{n} v\right)_{\mathbf{R}^{n}}= \\
& =\sup _{\|v\|_{V_{n}}=1}\left\|T_{n} v\right\|_{\mathbf{R}^{n}}=\left\|T_{n}\right\|_{V_{n} \rightarrow \mathrm{R}^{n}}=\frac{1}{\sqrt{v_{n}}} .
\end{aligned}
$$

Аналогично доказывается, что и

$$
\left\|\psi_{n}^{-1}\right\|_{\mathrm{R}^{n} \rightarrow V_{n}}=\left\|T_{n}\right\|_{V_{n^{\prime} \rightarrow \mathrm{R}^{n}}}=\frac{1}{\sqrt{\mu_{n}}} .
$$

При наложенных на $\Gamma_{n}$ ограничениях

$$
\left\|\psi_{n}^{-1} \Gamma_{n} T_{n}\right\|_{V_{n^{\prime}} \rightarrow V_{n}^{\prime}}<1 \quad \text { и } \quad\left\|\psi_{n}^{-1} \Gamma_{n} T_{n}\right\|_{H_{n} \rightarrow H_{n}}<1
$$

значит, операторы $I_{n}+\psi_{n}^{-1} \Gamma_{n} T_{n}$ обратимы в пространствах $\mathfrak{B}_{n}^{\prime}$ и $H_{n}$. Но тогда задача $(2.7)$, также как и система (1.7), имеет единственное решение.

По определению величины $c$ (1.5)

$$
\left\|h_{n}\right\| \leqslant c\left(\left\|h_{n}{ }^{\prime}+P_{n} A h_{n}\right\|_{\mathfrak{B}_{n}^{\prime}}+\left\|h_{n}(0)\right\|_{H_{n}}\right) .
$$

Отсюда в силу $(2.8),(2.9)$ и наложенных на $\Gamma_{n}$ и $\Delta_{n}(t)$ ограничений получаем оценку (2.1).

Для доказательства (2.2) возьмем $y_{n} \in V_{n}{ }^{\prime}$ такой, что $\left\|y_{n}\right\|_{V_{n}{ }^{\prime}}=1$, $\left\|T_{n} y_{n}\right\|_{\mathbf{R}^{n}}=\left\|T_{n}\right\|_{V_{n}{ }^{\prime} \rightarrow \mathrm{R}^{n}}$. $\quad$ Выберем также $x_{n} \in \mathbf{R}^{n}, \quad\left\|x_{n}\right\|_{\mathbf{R}^{n}}=1$, $\left\|\psi_{n}^{-1} x_{n}\right\|_{V_{n}{ }^{\prime}}=\left\|\psi_{n}^{-1}\right\|_{\mathrm{R}^{n} \rightarrow V_{n^{\prime}}} . \quad$ Нетрудно убедиться в существовании симметричной матрицы $\Gamma_{n}^{n}$ такой, что

$$
\Gamma_{n} T_{n} y_{n}=x_{n}, \quad\left\|\Gamma_{n}\right\|=\left\|x_{n}\right\|_{\mathrm{R}^{n}} /\left\|T_{n} y_{n}\right\|_{\mathrm{R}^{n}} ;
$$


ее можно умножить также на сколь угодно малое положительное число. Положим $u_{n}(t)=c_{n}(t) y_{n}$, где $c_{n}(t)-$ скалярная функция такая, что

$$
\left\|u_{n}{ }^{\prime}\right\|_{\mathfrak{B}_{n}{ }^{\prime}}=\left\|c_{n}{ }^{\prime}\right\|_{L^{2}(0, T)}=\left\|c_{n}\right\|_{L^{2}(0, T)}\left\|y_{n}\right\|_{V_{n}}=\left\|u_{n}\right\|_{\mathfrak{B}_{n}}
$$

Поскольку задача (1.4) задает равномерный по $n$ изоморфизм, то $\left\|\left|h_{n} \|\right|\right.$ оценима и в другом направлении, следовательно, при достаточно малой по норме $\Gamma_{n}$ мы получим оценку (2.2).

Оценки (2.3) и (2.4) доказываются аналогично, причем $\Delta_{n}$ (независимая от $t$ ) строится так же, как и $\Gamma_{n}$.

Для доказательства (2.5) и (2.6) выберем $g_{n}$ (независимый от $t$ ) и $\delta_{n}$, которые реализуют нормы $\left\|\psi_{n}^{-1}\right\|_{\mathbf{R}^{n} \rightarrow V_{n}^{\prime}} \quad$ и $\left\|\psi_{n}^{-1}\right\|_{\mathbf{R}^{n} \rightarrow H_{n}}$ соответственно.

Если в $(2.2),(2.3)$ или (2.4) $u_{n}$ построено, то положим $f_{n}(t)=$ $=u_{n}{ }^{\prime}(t)+P_{n} A(t) u_{n}(t)$ и выберем $f \in \mathfrak{S}^{\prime}$ (он всегда существует) такой, что $f_{n}=P_{n} f, \quad\left\|f_{n}\right\|_{\mathfrak{B}_{n}{ }^{\prime}}=\|f\|_{\mathfrak{B}^{\prime}}$. Положим также $\xi=u_{n}(0)$. Теорема доказана.

\section{3. Устойчивость}

Пусть выполнены условия (1.1) и (1.2).

$\mathrm{O}$ п р ед ел е н и е. Назовем метод Галеркина (1.4) для задачи (1.3) устойчивым в норме ||$\cdot \cdot \mid \|$, если существуют положительные $\gamma, r_{1}, r_{2}$ (не зависящие от $n$ и начальных данных $f$ и $\xi$ ) такие, что при $\left\|\Gamma_{n}\right\| \leqslant r_{1}$ и $\sup _{t \in[0, T]}\left\|\Delta_{n}(t)\right\| \leqslant r_{2}$ возмущенная задача (1.7) однозначно разрешима $t \in[0, T]$ и справедлива оценка

$$
\begin{gathered}
\left\|\widetilde{u}_{n}-u_{n}\right\| \leqslant \gamma\left(\left(\left\|\Gamma_{n}\right\|+\sup _{t \in[0, T]}\left\|\Delta_{n}(t)\right\|\right)\left(\|f\|_{\mathfrak{B}^{\prime}}^{2}+\|\xi\|_{H}^{2}\right)^{1 / 2}+\right. \\
\left.+\left\|g_{n}\right\|_{L^{2}\left(0, T ; \mathbf{R}^{n}\right)}+\left\|\delta_{n}\right\|\right),
\end{gathered}
$$

где $u_{n}$ и $\tilde{u}_{n}-$ решения приближенной (1.4) и возмущенной (2.7) задач.

След с тв и 1 . Необходимым и достаточным условием для устойчивости метода Галеркина в норме $\||\cdot|||$ является ограниченность последовательности $\mu_{n}$ снизу положительным числом.

Доказ ательство. Из условия inf $\mu_{n}>0$ следует сильная минимальность системы $\left\{w_{k}\right\}$ в $V^{\prime}$. Из этого, в свою очередь, вытекает сильная минимальность системы $\left\{w_{k}\right\}$ в $H$ и $V$, т. е. $\lim \lambda_{n}>0$ и $\lim v_{n}>0$. Поскольку $\left\|\left|u_{n}\right|\right\|$ оценивается равномерно по $n$ через $\left(\|f\|_{\mathfrak{B}^{\prime}}^{2}+\|\xi\|_{H}^{2}\right)^{1 / 2}, \quad$ a $\left\|u_{n}(0)\right\|_{H_{n}}$ - через $\|\xi\|_{H}$, из утверждения $1^{\circ}$ теоремы вытекает достаточность. Необходимость следует из (2.2) и утверждения $3^{\circ}$.

Следств и е 2. При условии inf $\mu_{n}>0$ имеет место устойчивость в норме пространства $C([0, T] ; H)$, т. е. справедлива оценка типа $(3.1)$, где норма $\|] \cdot \mid \|$ заменяется нормой $\|\cdot\|_{C([0, T] ; H)}$.

Пользуясь, например, теоремой вложения из $\left[{ }^{9}\right]$ (с. 33$)$, можно показать, что справедливо неравенство

$$
\begin{gathered}
\|v\|_{C\left([0, T] ; H_{n}\right)} \leqslant K\left(\|v\|_{L^{2}\left(0, T ; V_{n}\right)}^{2}+\left\|v^{\prime}\right\|_{L^{2}\left(0, T ; V_{n}{ }^{\prime}\right)}^{2}\right)^{1 / 2} \\
\left(v \in L^{2}\left(0, T ; V_{n}\right), v^{\prime} \in L^{2}\left(0, T ; V_{n}{ }^{\prime}\right)\right)
\end{gathered}
$$


где $K$ не зависит от $n$ и $v$. Утверждение следствия 2 вытекает из следствия 1 и неравенства (3.2).

С лед ств и е 3. Для координат $c_{n}(t)=\left(c_{n 1}(t), \ldots, c_{n n}(t)\right)$ и $\tilde{c}_{n}(t)=\left(\tilde{c}_{n 1}(t), \ldots, \tilde{c}_{n n}(t)\right)$ справедливы оценки

$$
\begin{gathered}
\left\|\tilde{c}_{n}-c_{n}\right\|_{L^{2}\left(0, T ; \mathbf{R}^{n}\right)} \leqslant \frac{1}{\sqrt{v_{n}}}\left\|\tilde{u}_{n}-u_{n}\right\|_{L^{2}\left(0, T ; V_{n}\right)}, \\
\left\|\tilde{c}_{n}{ }^{\prime}-c_{n}{ }^{\prime}\right\|_{L^{2}\left(0, T ; \mathbf{R}^{n}\right)} \leqslant \frac{1}{\sqrt{\mu_{n}}}\left\|\tilde{u}_{n}{ }^{\prime}-u_{n}{ }^{\prime}\right\|_{L^{2}\left(0, T ; V_{n}{ }^{\prime}\right)}, \\
\left\|\tilde{c}_{n}-c_{n}\right\|_{C\left([0, T] ; \mathbb{R}^{n}\right)} \leqslant \frac{1}{\sqrt{\lambda_{n}}}\left\|\tilde{u}_{n}-u_{n}\right\|_{C\left([0, T] ; H_{n}\right)}
\end{gathered}
$$

(нужно лишь заметить, что $\tilde{c}_{n}-c_{n}=T_{n}\left(\tilde{u}_{n}-u_{n}\right)$ ), значит, при условии inf $\mu_{n}>0$ имеет место устойчивость координат в приведенных нормах.

Отметим, что $K$ в неравенстве (3.2) не увеличивается с возрастанием $T$, поэтому, если предположить, что условия (1.1) и (1.2) выполнены для $t \in[0, \infty)$ и $f \in L^{2}\left(0, \infty ; V^{\prime}\right)$, то полученные результаты можно распространить на бесконечный промежуток.

Существуют примеры, доказывающие, что из сильной минимальности системы $\left\{w_{k}\right\}$ в $V^{\prime}$ не следует условие inf $\mu_{n}>0$.

Оценки (2.1) - (2.6) показывают, что особое внимание следует уделять вычислению скалярных произведений $\left(w_{k}, w_{i}\right)$. Если они вычисляются точно, то метод Галеркина будет устойчивым в норме $\||\cdot|\|$ при условии $\lim \lambda_{n}>0$. Такое положение имеет место, например, если $\left\{w_{k}\right\}$ - ортонормированная в $H$ система.

3 а м е ч а н и е. Предположим, что $A(t)$ - самосопряженный, положительно определенный в $H$ оператор, область определения которого не зависит от $t$, и что существует сильная производная $A^{\prime}(t)$. Если положить $V=H_{A(0)}$, будут выполнены условия (1.1) и (1.2). Допустим далее, что $A^{\prime}(t)$ неположительна, $f \in L^{2}(0, T ; H)$. Тогда при условии $\lim \lambda_{n}>0$ (это более слабое требование, чем inf $\mu_{n}>0$ ) удается несколько усилить и расширить результаты работ $\left[{ }^{1-4,6-8}\right]$, где доказывается устойчивость в норме $C([0, T] ; H)$. Например, устойчивость метода Галеркина устанавливается в более сильных нормах: величина

$$
\left(\int_{0}^{T}\left(\left\|h_{n}^{\prime}(t)\right\|_{H}^{2}+\left\|P_{n} A(t) h_{n}(t)\right\|_{H}^{2}\right) d t\right)^{1 / 2}+\max _{t \in[0, T]}\left\|A^{1 / 2}(t) h_{n}(t)\right\|_{H}
$$

оценивается сверху через все возмущения и снизу через отдельные возмущения подобно $(2.1)-(2.6)$, значит, здесь условие $\lim \lambda_{n}>0$ является и необходимым. При почти ортонормированности системы $\left\{w_{k}\right\}$ в $H$ результаты работы $\left[{ }^{5}\right]$ справедливы также для переменного оператора.

\section{ЛИТЕРА Т Р А}

1. Михлин С. Г., Численная реализация вариационных методов, М., 1966.

2. В елли е М. А., Докл. АН СССР, 157, № 1, 16 (1964); поправка: Докл. АН CССР, 161, № 2, 262 (1965)

3. В ели е в М. А., Сиб. матем. ж., 9, № 4, 783 (1968).

4. Ве ли е в М. А., Дифференциальные уравнения, 5, № 3, 479 (1969).

5. Ицик Б. Г., Соболевски й П. Е., Тр. НИИ матем. ВГУ, 2, 39 (1970).

6. Тополянский Д. Б., З ап руд ски й Я. М., Днфференциальные уравнения и их приложения, Днепропетровск, 113 (1971). 
7. Тополянский Д. Б., З а прудский Я. М., Вычислительная и прикладная математика, 18, 40 (1972).

8. Тополянский Д. Б., З З прудский Я. М., Укр. матем. ж., 26, № 5, 621 (1974).

9. Л ионс Ж.-Л., М ад женес Э., Неоднородные граничные задачи и их приложения, М., 1971.

10. В а й ни к ко Г. М., О я П. Э., Дифференциальные уравнения, 11, № 7, 1269 (1975)

11. Красносельский М. А. и др., Приближенное решение операторных уравнений, М., 1969.

Тартуский государственный университет

Поступила в редакцию

$24 /$ XII 1975

P. OJA

\section{GALJORKINI MEETODI STABIILSUSEST EVOLUTSIOONIVORRANDITE KORRAL}

Uuritakse Galjorkini meetodi stabiilsust ülesande $u^{\prime}+A(t) u=f(t), u(0)=\xi$ lahendamisel eeldustel, mille korral J.-L. Lions on tõestanud lahendi olemasolu ja ühesuse. Selleks hinnatakse täpse ja praktilisel lahendamisel saadava ligikaudse Galjorkini lähendi vahet kahepoolselt vigade kaudu, mis tekivad Galjorkini võrrandis esinevate skalaarkorrutiste ligikaudsel arvutamisel. Galjorkini lähendi häiritust hinnatakse normides, mille korral on tõestatud meetodi koonduvus. Hinnangutest saadakse meetodi stabiilsuse tarvilik ja piisav tingimus.

\section{P. OJA}

\section{ON STABILITY OF THE GALERKIN METHOD FOR EVOLUTION EQUATIONS}

The object of the present paper is to investigate stability of the Galerkin method for the initial value problem for the evolution equation $u^{\prime}+A(t) u=f(t), u(0)=\xi$. The results are obtained in the case in which the existence and uniqueness of the solution is proved by J.-L. Lions. The influence of the perturbations appearing in a practical realization of the Galerkin equations, on the approximate solutions is characterized by two-way estimates in the same norms in which the convergence is proved. Necessary and sufficient conditions are given to the stability of the Galerkin method. 